\title{
VEGF expression in mesenchymal stem cells promotes bone formation of tissue-engineered bones
}

\author{
BOLING LIU ${ }^{1}$, XIHAI LI ${ }^{2}$, GUIQING LIANG ${ }^{1}$ and XIANXIANG LIU ${ }^{2}$ \\ ${ }^{1}$ Fuzhou Integrated Chinese and Western Medicine Hospital of Fujian Province, Fuzhou 350007; \\ ${ }^{2}$ Academy of Integrated Chinese and Western Medicine of Fujian University of \\ Traditional Chinese Medicine, Fuzhou, Fujian 350108, P.R. China
}

Received April 27, 2011; Accepted August 9, 2011

DOI: $10.3892 / \mathrm{mmr} .2011 .559$

\begin{abstract}
The purpose of this study was to investigate the in vivo vascularization and bone formation activity of tissue-engineered bone constructed using bone marrow mesenchymal stem cells (MSCs) transfected with vascular endothelial growth factor (VEGF). The expression of VEGF165 in rat bone marrow MSCs was confirmed using RT-PCR and immunohistochemistry. The MSCs were cultured together with nano-hydroxyapatite/collagen (NHAC) to form tissue-engineered bone. Untransfected MSCs were used as controls. The mice were sacrificed, and the bone xenografts were analyzed using immunohistochemistry and quantified for the degree of vascularization and new bone formation. Based on our results, expression of the VEGF165 gene was detected using RT-PCR and immunohistochemistry following transfection and 4 weeks of selection. The co-cultured NHACand VEGF-transfected MSCs had significantly higher alkaline phosphatase (AP) activity compared to the controls $(\mathrm{P}<0.05)$. In the mice that received the tissue-engineered bone xenografts, clumps of cartilage cells, irregular bone-like tissue and microvessels were observed. The growth of these structures progressed with time. In the control mice, however, only small amounts of bone-like and fibrotic tissue were observed. The differences between the control and experimental groups were statistically significant $(\mathrm{P}<0.05)$. In conclusion, VEGF165-transfected bone marrow MSCs promotes vascularization of tissue-engineered bone and ectopic osteogenesis.
\end{abstract}

\section{Introduction}

Bone damage, osteonecrosis, and other types of bone loss are common problems faced by orthopedic specialists. Currently,

Correspondence to: Professor Xianxiang Liu, Academy of Integrated Chinese and Western Medicine of Fujian University of Traditional Chinese Medicine, 1 Huatuo Road, University Town, Minhou Shangjie, Fuzhou, Fujian 350108, P.R. China

E-mail: liuxianxiang@163.com

Key words: vascular endothelial growth factor, bone marrow mesenchymal stem cells, tissue-engineered bone, vascularization, ectopic osteogenesis application of tissue engineering techniques to repair bone loss is a therapeutic development trend (1). Vascularization of tissueengineered bone has become a primary focus of research in this area. In the process of bone rebuilding, an intact vessel network should be constructed to support correct signaling between cells and the extracellular matrix, provide an appropriate environment for bone growth and eventually lead to the formation of functional tissue-engineered bone (2). The currently used tissue-engineered bone, which is composed of frame material, seed cells, and regulating factors, is markedly limited by the lack of vessel networks and appropriate nutrients in the xenograft (3). In vitro-cultured bone complexes of cells and frame material obtain nutrients and oxygen primarily through infiltration and diffusion. Following transplantation of the cultured bone complex, small vessels from peripheral tissue gradually grow into the small bone complex and connect it with the peripheral tissue to supply nutrients. However, the cells far from the small vessels originating in the surrounding tissue are unable to survive due to the lack of nutrients and oxygen (4). In addition, if the transplanted areas suffer from malcirculation, it may take a longer period of time for the xenograft to vascularize, leading to impaired cell proliferation, differentiation and even cell death. Subsequently, defects in vascularization may lead to failure of the transplanted bone. Therefore, the blood supply should be established as early as possible. Promoting vascularization may also facilitate greater access by bone-stimulating cytokines to enhance the rapid growth of the bone xenograft (5).

Vascular endothelial growth factor (VEGF) is a specific growth factor that promotes the proliferation and chemotaxis of endothelial cells. It has been shown to regulate osteogenesis, bone development and regeneration (6,7). In recent years VEGF has increasingly been used to prevent or treat ischemic diseases (8). It has been hypothesized that if VEGF were applied in tissue-engineered bone, the vascularization of the bone xenograft may be improved. Exogenously-applied VEGF usually has a short half-life and is rapidly diluted and metabolized when used locally. The requirement for high dosages and a high frequency of doses is not cost-effective. We utilized transfection to express VEGF protein in seed cells and bone complexes constructed with these cells, facilitating local secretion of the VEGF protein and promotion of vascularization of the bone xenograft, to determine an alternative method of enhancing the vascularization of tissue-engineered bone. 


\section{Materials and methods}

Reagents. The eukaryotic expression vector for human VEGF165, pcDNA3.1-hVEGF165, was kindly provided by the Jiangsu Province Blood Research Institute. The reagents used in this study included: pcDNA3.1 plasmid, fetal bovine serum (FBS), Dulbecco's modified Eagle's medium (DMEM), trypsin, lipofectamine and G418 from Invitrogen Inc. (Carlsbad, CA, USA); glycerol 2-phosphate disodium, vitamin C, dexamethasone, anti-CD45 antibody and anti-CD90 antibody from Sigma-Aldrich (St. Louis, MO, USA); type IV collagenase from Worthington (Lakewood, NJ, USA); rabbit anti-human VEGF immunohistochemistry kit from Maixin Bio. (Fuzhou, Fujian, China); FITC-labeled secondary antibodies from Boster Inc. (Wuhan, Hubei, China); osteocalcin (OCN) radioimmunometric kit from Beijing ChemClin Biotech. (Beijing, China); and alkaline phosphatase (AP) activity kit from Jiancheng Biotech. (Nanjing, Jiangsu, China). The nano-HAP/ Collagen (NHAC) (provided by the Department of Material, Tsinghua University, China) was formed into $5 \times 5 \times 5 \mathrm{~mm}$ and decontaminated using $\mathrm{C}^{60}$ irradiation.

Animals. Sprague-Dawley (SD) rats at 6 weeks (each weighing 100-110 g) and 6-week-old nude mice (each weighing 18-26 g) were purchased from Super-BK Laboratory Animal Inc. (Shanghai, China).

Isolation, culture and identification of rat bone marrow mesenchymal stem cells (MSCs). Healthy SD rats were sacrificed by cervical dislocation, and the tibia and femur were excised under sterile conditions. The bones were submerged in 75\% ethanol for 3-5 min, and the bone marrow cells were flushed out with DMEM medium supplemented with $15 \%$ FBS. The cells were mixed 1:1 with Percoll lymphocytes isolation solution, centrifuged to isolate the write ring and washed twice. The cells were resuspended at $1 \times 10^{6} / \mathrm{ml}$ in DMEM with $15 \% \mathrm{FBS}$, and incubated in $50 \mathrm{ml}$ flask at $37^{\circ} \mathrm{C}$ and $5 \% \mathrm{CO}_{2}$. The cells were labeled as F0. The culture medium was changed every $72 \mathrm{~h}$. The cells were observed once per day and passaged on reaching $90 \%$ confluence. The F2 cells were trypsinized, incubated with monoclonal antibodies anti-CD45-FITC and CD90-FITC at room temperature for $25 \mathrm{~min}$, and washed with PBS. The positive rate for the surface markers CD45 and CD90 was detected using fluorescence activated cell sorting (FACS).

Transfection of VEGF into MSCs. Following passage 2, MSCs were seeded in 6-well plates and after reaching 50-80\% confluence the cells were transfected with $1 \mu \mathrm{g}$ of pcDNA3.1VEGF165 or pcDNA3.1 empty vector in serum-free medium using lipofectamine according to the manufacturer's protocol. The medium was changed after $6 \mathrm{~h}$ and the cells were further cultured for $48 \mathrm{~h}$ prior to the addition of G418. After 10-12 days, the cell colonies were visible and selected at random. The cell clones were cultured in bone conditional medium (DMEM with 15\% FBS, $10 \mathrm{mM}$ glycerol 2-phosphate disodium, $50 \mathrm{mg} / 1$ vitamin $\mathrm{C}$ and $10 \mathrm{nM}$ dexamethasone) to expand the cells. After further culturing for 4 weeks, the cells transfected with pcDNA3.1-VEGF165 and pcDNA3.1, or untransfected cells were tested for VEGF expression using RT-PCR and immunostaining (rabbit anti human VEGF immunohistochemistry kit). The PCR primers used were: P1: 5'-GAATTCTCGGGCCTCCGAAACCATGAAC-3', and P2: 5'-AAGCTTTCACCGCCTCGGCTTGTCACAT-3'.

Co-culture of MSCs and NHAC. The decontaminated NHAC was divided into 2 groups: the experimental culture with $\mathrm{F} 2$ (secondary passage cells) VEGF-expressing MSCs, and the control culture with untransfected MSCs. At 1 day prior to co-culture, NHAC was incubated in DMEM for $8 \mathrm{~h}$ and airdried in the hood. The cells were resuspended at $1 \times 10^{6} / \mathrm{m} 1$ and added to NHAC dropwise to allow the cells to penetrate the NHAC completely. The complexes were incubated at $37^{\circ} \mathrm{C}$ and $5 \% \mathrm{CO}_{2}$ in a humidified incubator. After $8 \mathrm{~h}$, the medium was added. At 3 and 6 day-incubation, the bone complexes were examined using a scanning electron microscope and used in mouse xenograft experiments.

Identification of the MSCs-NHAC complexes. The MSC-NHAC complex was identified by measuring $\mathrm{OCN}$ secretion and $\mathrm{AP}$ activity according to the manufacturers' protocols. Briefly, the culture medium was collected and lyophilized 6 days after culture. The media from the experimental and control groups were resolubilized. Together with the OCN standards, the samples of resuspended media were incubated with an antiOCN antibody and ${ }^{125}$ I followed by scintillation counting. The amount of OCN in the medium was calculated according to the OCN standards.

To measure the activity of AP, the complexes from the 2 groups were digested using $2.5 \mathrm{~g} / \mathrm{l}$ trypsin, and the dissociated cells were washed out with PBS, centrifuged at $150 \mathrm{rcf}$ at $4^{\circ} \mathrm{C}$, and washed with PBS 3 times. After the last wash, the cells were resuspended in $200 \mu$ l of PBS, lysed by 3 freezethaw cycles, and analyzed for AP activity using the kit.

Transplantation of the bone grafts. Healthy nude mice were randomly divided into control and experimental groups $(n=8)$. The mice were anesthetized with $0.5 \%$ ketamine hydrochloride and ethoxyethane. The skin was then cut open along the middle back line. Sections of MSC-NHAC complexes cultured for 3 days with VEGF-expressing MSCs (i.e., VEGF-expressing bone grafts) were bilaterally transplanted under the skin of nude mice in the experimental group. The nude mice in the control group were bilaterally transplanted with MSC-NHAC complexes cultured for 3 days with untransfected MSCs (i.e., control bone grafts). Following, surgery, the mice were maintained in sterile conditions in separate cages. At 4 and 8 weeks postoperatively, 4 mice in each group were anesthetized and sacrificed, and the bone xenografts were excised. A total of 8 samples (4 nude mice, each sample repeated for 2 times) in each group were paraffin-sectioned, and analyzed using H\&E and Masson stainings and microscopy. Four H\&E staining slides across the center of the 4 samples for each time point in the 2 groups were analyzed using a XTH-/VTV Microscopic Image Analysis System to analyze the microvessel. After image acquisition, import and export, the individual field was divided into 1024 statistical area units (u) and the area occupied by vessels was calculated to evaluate the vascularization of bone grafts. Map info software (Mapinfo, Troy, NY, USA) was used to measure the surface area of the newly formed bone. Five fields were recorded for each slide and the result 

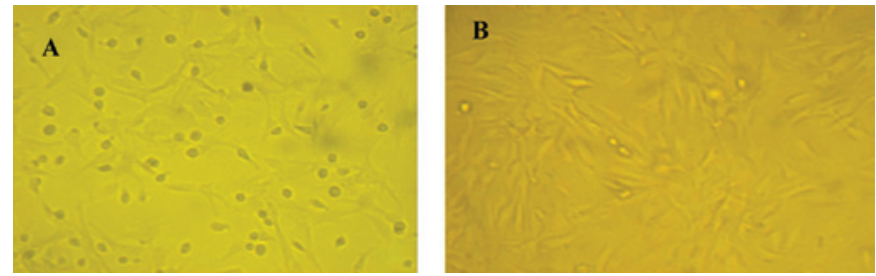

Figure 1. Morphology of primary and passaged MSCs (magnification, x100). (A) Image of cells cultured for 9-11 days. (B) Passage 1 MSCs after culture for 3-4 days.

was calculated as the ratio of the new bone surface area to the total filled area.

Statistical analysis. Statistical data are expressed as the mean \pm SD. Statistical analysis was performed by one-way analysis of variance (ANOVA). Differences were considered statistically significant when $\mathrm{P}<0.05$.

\section{Results}

Morphology and characterization of the rat bone marrow MSCs. The primary MSCs were spherical with good refractivity and exhibited a diffuse distribution in the culture flask immediately after being seeded. After $24 \mathrm{~h}$, a small fraction of the cells started to adhere and exhibited a large square or oval morphology. After $48 \mathrm{~h}$, a greater number of single nucleus cells had adhered, and certain MSCs exhibited a spindle or polygonal morphology. After 5-8 days, the MSCs gradually became isolated colonies with different shapes and variable volumes. After 9-11 days, the MSCs showed marked proliferation, and the colonies began to fuse together (Fig. 1A). After 14-21 days, the MSCs were split for further culture. The passaged cells primarily exhibited spindle morphology and were evenly distributed (Fig. 1B). The cells were passaged every 3-4 days thereafter. The expression of CD45 and CD90 in the MSCs was examined by FACS (Fig. 2). Our results confirmed that the positive rate was $2.14 \%$ for CD45 and $99.12 \%$ for CD90.

Transfection of MSCs and expression of VEGF. Isolated rat MSCs were transfected with an hVEGF165 expression vector using lipofectamine. The expression of hVEGF165 was initially examined using RT-PCR. As shown in Fig. 3, an amplified product of $604 \mathrm{bp}$ was clearly visible on the agarose gel when the cDNA from cells transfected with pcDNA3.1VEGF165 was used as the template. In contrast, no product was amplified from pcDNA3.1 empty vector-transfected or untransfected MSCs, suggesting that the plasmid pcDNA3.1VEGF165 were successfully transfected into the rat MSCs, and that the hVEGF mRNA was transcribed. Expression of hVEGF protein was further validated using immunohistochemistry. The results revealed that hVEGF was positively stained in pcDNA3.1-VEGF165-transfected cells; however, no staining was observed in empty vector-transfected or untransfected cells (Fig. 4).

Construction of the MSCs-NHAC complex. Following co-culture of the MSCs and NHAC material, the complex was first observed under inverted microscopy. Since the NHAC
A

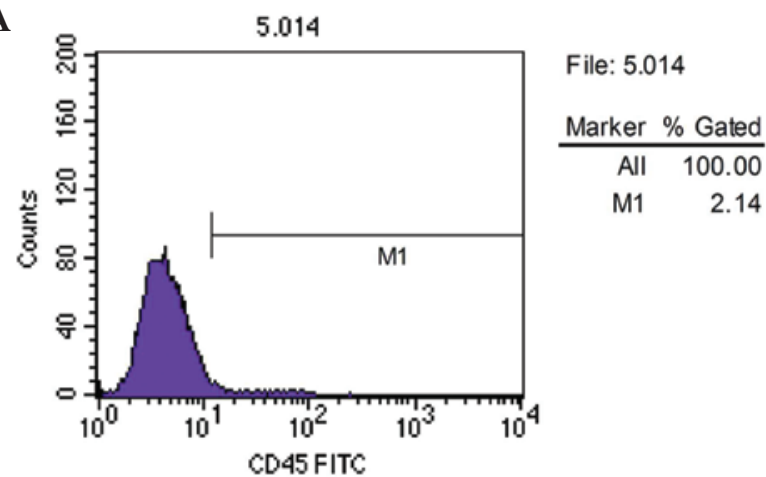

B

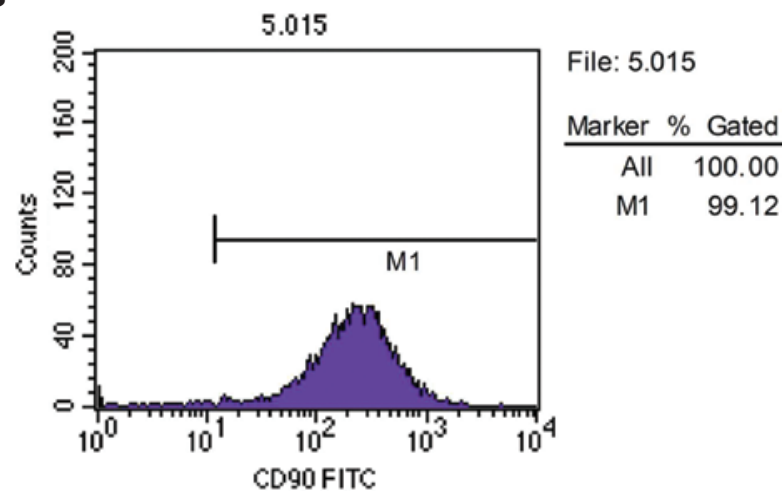

Figure 2. (A) FACS of cells stained with anti-CD45 antibody. (B) FACS of cells stained with anti-CD90 antibody.

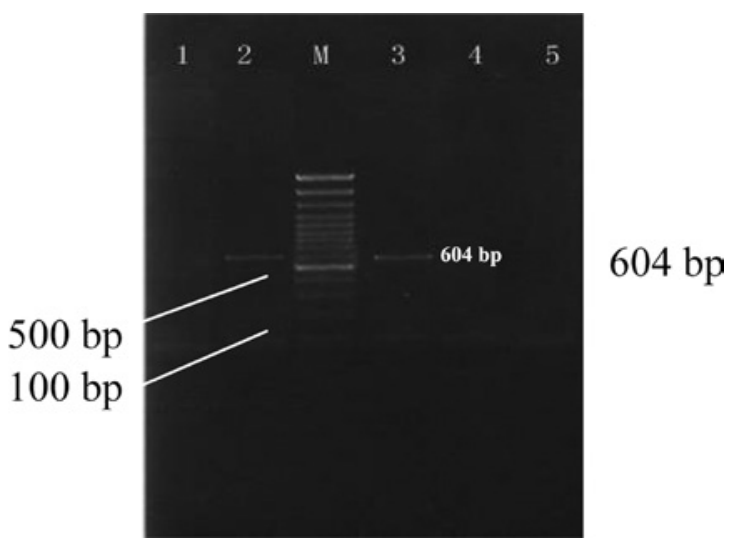

Figure 3. Identification of the transfected hVEGF using RT-PCR (A) RT-PCR amplification of hVEGF from cells transfected with different plasmids: Lane 1, pcDNA3.1 vector-transfected cells at 4 weeks; lane 2, pcDNA3.1-hVEGF165-transfected cells at 4 weeks; lane M, DNA marker; lane 3, pcDNA3.1-hVEGF165-transfected cells at $48 \mathrm{~h}$; lane 4, pcDNA3.1 vector-transfected cells at $48 \mathrm{~h}$; lane 5 , untransfected cells.
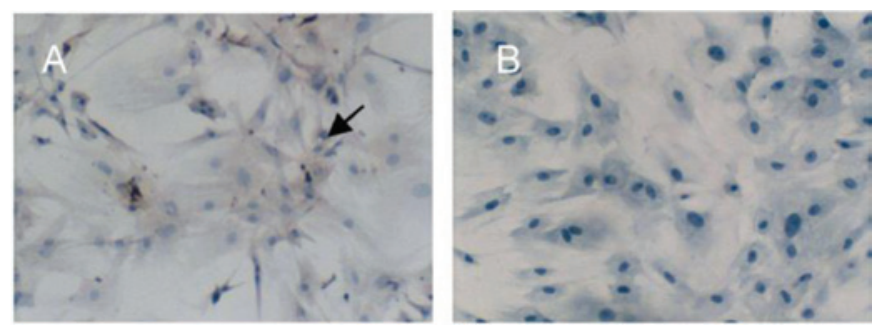

Figure 4. Immunostaining of the MSCs (magnification, x100). (A) hVEGF staining of the pcDNA3.1-hVEGF165 transfected cells. (B) hVEGF staining of the pcDNA3.1 empty vector-transfected cells. 


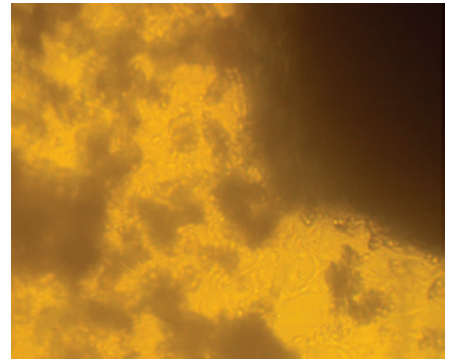

Figure 5. MSCs-NHAC complex after co-culture for 3 days (inverted microscopy; magnification, $\mathrm{x} 100)$.

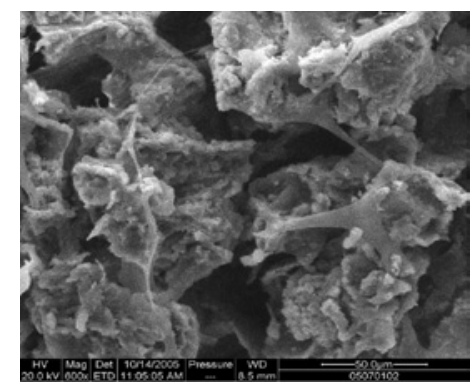

Figure 6. MSCs-NHAC complex after co-culture for 3 days (scanning electronic microscopy; magnification, $\mathrm{x} 200$ ).
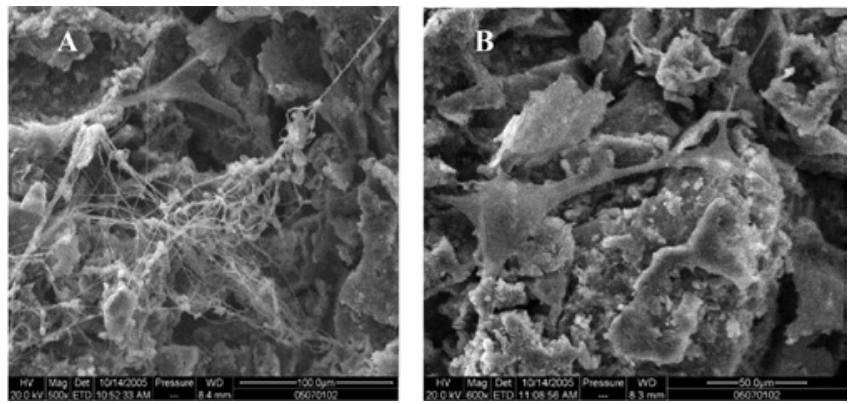

Figure 7. The MSCs-NHAC complex after co-culture for 6 days (scanning electronic microscopy; magnification, x200). (A and B) Experimental and control groups, respectively.

material was opaque, the cells on the surface of the NHAC particles were difficult to observe by microscopy. Only MSCs attached to the edges of the NHAC particles were observed. After extension of the culture time, the MSCs on the edge and bottom of the NHAC particles increased gradually, although the distribution was uneven, with more cells attached to the bottom of the particles (Fig. 5).

The MSCs-NHAC complexes were then examined using scanning electronic microscopy. The NHAC particle exhibited a porous 3-dimensional network with numerous ordered and connected holes. A monolayer of cells with varying morphologies and multiple extrusions was present on the surface and in the holes of the NHAC particles (Fig. 6). Floc-like, wellconnected extracellular matrices were observed on the surface and between the cells in the experimental group [ECM similar to collagen (Fig. 7A)]. Little or no secreted ECM was evident in the control group (Fig. 7B). No marked adherent or morphological differences between the cells in the control and the experimental groups.
Table I. OCN concentration and the AP activity in the MSCsNHAC complexes (mean $\pm \mathrm{SD}$ ).

\begin{tabular}{lcc}
\hline Group & $\begin{array}{c}\text { OCN concentration } \\
(\mathrm{ng} / \mathrm{ml})\end{array}$ & $\begin{array}{c}\text { AP activity } \\
(\mathrm{u} / 100 \mathrm{ml})\end{array}$ \\
\hline Experimental & $2.23 \pm 0.08^{\mathrm{a}}$ & $23.95 \pm 0.40^{\mathrm{a}}$ \\
Control & $1.27 \pm 0.05$ & $17.70 \pm 0.81$ \\
\hline
\end{tabular}

${ }^{a} \mathrm{P}<0.05$ compared with the control group. OCN, osteocalcin; $\mathrm{AP}$, alkaline phosphatase.
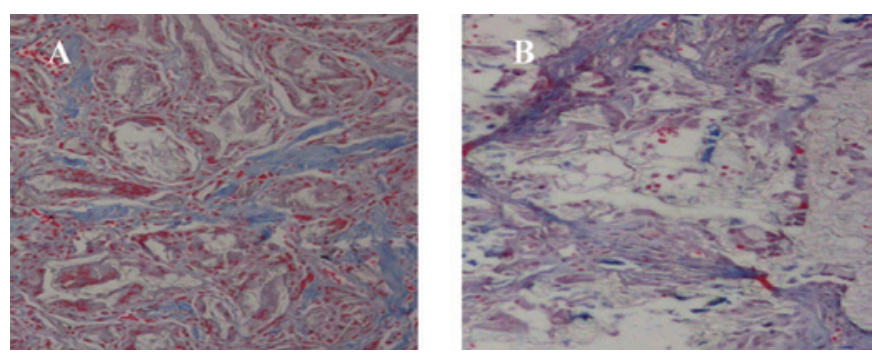

Figure 8. Masson staining of the MSCs-NHAC xenograft 4 weeks after transplantation (magnification, x100). (A) Experimental group. The material is partially dissolved and absorbed. Primary irregular bone-like tissue is shown to penetrate into the holes along with the microvessels. There are abundant microvessels and the degree of calcification of the newly-formed bone tissue was relatively low. (B) Control group. Some cartilage-like and bone-like tissues are visible with few microvessels.
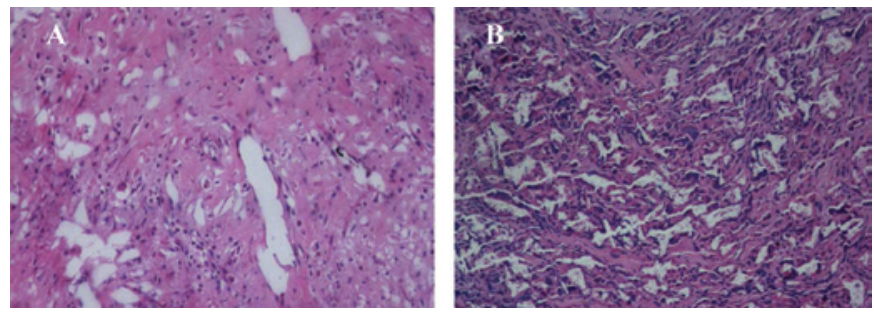

Figure 9. Masson staining of the MSCs-NHAC xenograft 8 weeks after transplantation (magnification, $\mathrm{x} 80$ ). (A) Experimental group. The material is partially dissolved and absorbed. Large amounts of mature woven bone and some cartilage-like tissue are visible in the edge area. Along with the appearance of microvessels, the vessel networks began to be well-organized. (B) Control group. There are some cartilage-like and bone-like tissues with few microvessels and fibers.

OCN and AP activity of the MSC-NHAC complex. We also measured the concentration of OCN and the activity of the AP in the MSC-NHAC complex. The results are shown in Table I.

Histology, vasculaturization, and new bone formation in the xenografts. The spaces of the unabsorbed and decalcified NHAC were visible in all slides, among which there was new bone tissue growing. At 4 weeks after transplantation, there were large numbers of clumps of cartilage cells. Irregular bone-like tissue was found to penetrate the holes of NHAC along with microvessels, which were interconnected to form vascular anastomosis and complex network. The degree of calcification of the newly-formed bone tissue was relatively low. The vasculature was more abundant in the experimental 
Table II. Quantitation of the vascularization at various time points and groups (using the XTH-/VTV Microscopic Image Analysis System).

\begin{tabular}{lcc}
\hline Group & 4 weeks & 8 weeks \\
\hline Experimental & $308.25 \pm 3.89^{\mathrm{a}}$ & $222.17 \pm 3.66^{\mathrm{a}}$ \\
Control & $216.08 \pm 3.70$ & $159.50 \pm 5.74$ \\
\hline
\end{tabular}

After image capture, import and export, the individual field was divided into 1024 statistical area units (u) and the area occupied by vessels was calculated to evaluate the vascularization of bone grafts. $(\mathrm{n}=8$, mean $\pm \mathrm{SD}) .{ }^{\mathrm{a}} \mathrm{P}<0.05$ compared with the control group.

Table III. Quantitation of the new bone formation at various time points and groups (measured by the area percentage of the new bone). ${ }^{\mathrm{a}}$

\begin{tabular}{lcc}
\hline Group & 4 weeks & 8 weeks \\
\hline Experimental & $30.26 \pm 1.43^{\mathrm{b}}$ & $50.35 \pm 2.56^{\mathrm{b}}$ \\
Control & $18.58 \pm 1.02$ & $35.75 \pm 2.18$ \\
\hline
\end{tabular}

${ }^{\mathrm{a}}(\mathrm{n}=8$, mean $\pm \mathrm{SD} \%) .{ }^{\mathrm{b}} \mathrm{P}<0.05$ compared with the control group.

group compared to the control group (Fig. 8). At 8 weeks after transplantation, we observed high levels of mature woven bone and some cartilage-like tissue. Microvessels were also formed. The vessel networks were well-organized, and regularly and evenly distributed. The difference of blood vessel diameters was small although the vessels were arranged relatively loosely. In the control group, there was significantly less bone-like and cartilage-like tissue with few microvessels and fibers (Fig. 9).

Vascularization and bone formation. We quantitatively analyzed the vascularization of the complex xenografts, and the results are shown in Table II. The vessel areas in the 4 and 8 weeks bone grafts of the experiment group were significantly higher compared to those of the control group $(\mathrm{P}<0.05)$. The vessel areas of 8 -week bone grafts in the experimental and control groups were lower compared to those of 4-week bone grafts, indicating that the bone grafts may have demonstrated revascularization. The quantitation of bone formation was also analyzed and is shown in Table III. The new bone formation in the 4- and 8-week bone grafts of the experimental group was significantly higher compared to that in those of the control group $(\mathrm{P}<0.05)$, indicating that the VEGF transfection and expression in bone grafts is capable of promoting new bone formation after implantation.

\section{Discussion}

There are three basic stages following bone transplantation: vascularization of the transplant, bone regeneration, and fusion of the ends. Of these, vascularization is of particular significance, since its function is required for and directly determines the efficacy of the following two steps. The infiltration of the microvessels conveys the osteoblasts, nutrients, growth factors and other related cells into the microenvironment around the repaired bone. The vessels also remove degraded materials and metabolic byproducts to maintain a favorable metabolic environment for bone regeneration (9). To repair the damaged bone with tissue-engineered bone also requires vascularization. After in vitro co-culture of the frame material and the seed cells, the tissue-engineered bone was able to be constructed and implanted. Following transplantation, the implant was rapidly vascularized to obtain nutrients, maintain viability, and perform its function to reconstruct the local bone structure.

The MSCs are relatively easily obtained from multiple sources and are ideal seed cells for tissue engineering $(10,11)$. They also proliferate robustly and are easily transfected with exogenous genes, rendering them suitable for bone growth factor-based gene therapy (12). Although an MSC may follow multiple differentiation pathways, the differentiation is not a spontaneous process. Cytokines and their environment in vivo may promote a specific differentiation path. VEGF is the specific growth factor that enhances the proliferation and chemotaxis of endothelial cells and is a critical regulator of development and regeneration of bone (13). A number of studies have reported the application of VEGF in promoting new vasculature formation. Gene therapy using VEGF may promote collateral circulatory development and increase blood volume in an ischemic limb (13). The serum containing VEGF165 not only promotes the proliferation and differentiation of endothelial progenitor cells (EPCs), but also stimulates EPCs to become involved in vessel formation (14). The purpose of gene therapy is to introduce specific genes into cells in order that the gene product can be synthesized and/or secreted (15). Following transfection with the target gene, the cells may produce the target growth factor, which in turn binds to the receptors on the same cells and peripheral cells. In this manner, the biological effects of the growth factor may be enhanced through auto- and paracrine signaling (16). In addition, the gene product produced by the cells has undergone post-translational modifications and may possess greater biological activity than the exogenous proteins (17). In our study, we transfected the hVEGF165 gene into MSCs using a standard liposome-mediated technique and validated the expression and secretion of VEGF by RT-PCR and immunohistochemistry.

After in vitro co-culture of MSCs and the frame material, both the transfected and untransfected cells grew favourably, as examined using inverted microscopy. Under scanning electronic microscopy, we observed that the two groups of cells adhere, grow, proliferate and infiltrate into the holes of the NHAC particles, suggesting that the NHAC material is bio-compatible and harmless to the cells. The cells in the experimental group (transfected with VEGF165) secrete ECM-associated molecules including collagen and calcium crystal, whereas the cells in the control (untransfected) group secrete significantly lower amounts of ECM. Our results underscore the importance of VEGF in bone tissue growth, which has been the subject of numerous previous studies. Midy and Plouet found that the AP and $\beta$-cAMP concentration in in vitro-cultured osteoblasts was elevated 4-fold following exogenous VEGF treatment (18). Mayer et al and Geiger et al also revealed that VEGF improved the activity of osteoblasts and enhanced migration, proliferation and differentiation 
$(19,20)$. In addition, it has been shown that VEGF is involved in antagonizing apoptosis and maintaining survival of osteoblasts $(5,21)$. AP activity is a significant biomarker of osteoblasts and is critically involved in metabolism and bone mineralization. The enzymatic activity of AP and the accumulation of ECM provide a suitable $\mathrm{pH}$ and calcium/phosphate concentration, which significantly improves the bone-formation activity of MSCs. OCN accounts for 10-20\% of total non-collagen proteins in bone and is the specifically secreted product of osteoblasts. Levels of this protein reflect the bone-formation activity of the cells. In our study, after 6 days of culture with the NHAC material, the experimental group cells exhibited significantly higher AP activity and OCN concentration than the control group, indicating that hVEGF165 expression promotes the transition of MSCs to osteoblasts.

The model for ectopic osteogenesis possesses the morphological structures and metabolic features of osteogenesis, including bone trabecula and cavum medullare ossium. The model excludes interference by the local environment during the repair of the damaged bone, and more efficiently reflects the bone-formation activity and metabolic processes of the xenograft (22). Following transplantation of the tissue-engineered bone into the subcutaneous tissue of nude mice, the vascularization and mature bone formation of the experimental group were found to be superior to those of the control group at each time point, using both morphological and quantitative assessments. However, we found that the vessel areas of 8-week bone grafts in the experiment and control groups were lower than those of the 4-week bone grafts. Morphologically, the vessel networks at 8 weeks were well-organized and regularly and evenly distributed. The difference of blood vessel diameters was small although the vessels were arranged relatively loosely. This phenomenon may have been caused by the rebuilding and redistribution of newly formed vessels in the bone grafts.

In conclusion, our results reveal that the tissue-engineered bone constructed using hVEGF165-transfected MSCs and NHAC material demonstrated increased revascularization and bone-formation activity compared to the controls.

\section{Acknowledgements}

This study was supported by the Open Fund of Fujian Key Laboratory of Integrative Medicine on Geriatrics (no. 2008J1004-16) and the Development Fund of Chen Keji Integrated Chinese and Western Medicine (no. CKJ2008003). The authors thank Dr Xia Bai from the Jiangsu Province Blood Research Institute for providing the hVEGF165 fulllength cDNA expression vector pcDNA3.1-hVEGF165 and Professor Qingling Feng from the Tsinghua University for providing the NHAC material.

\section{References}

1. Pietrzak WS, Woodell-May J and McDonald N: Assay of bone morphogenetic protein-2, -4 , and -7 in human demineralized bone matrix. J Craniofac Surg 17: 84-90, 2006.

2. Patel ZS and Mikos AG: Angiogenesis with biomaterial-based drug- and cell-delivery systems. J Biomater Sci Polym Ed 15: 701-726, 2004.

3. Griffith CK, Miller C, Sainson RC, Calvert JW, Jeon NL, Hughes CC and George SC: Diffusion limits of an in vitro thick prevascularized tissue. Tissue Eng 11: 257-266, 2005.
4. Kidd KR, Nagle RB and Williams SK: Angiogenesis and neovascularization associated with extracellular matrix-modified porous implants. J Biomed Mater Res 59: 366-377, 2002.

5. Street J, Bao M, De Guzman L, Bunting S, Peale FV Jr, Ferrara N, Steinmetz H, Hoeffel J, Cleland JL, Daugherty A, et al: Vascular endothelial growth factor stimulates bone repair by promoting angiogenesis and bone turnover. Proc Natl Acad Sci USA 99: 9656-9661, 2002.

6. Ferrara N: Vascular endothelial growth factor and the regulation of angiogenesis. Recent Prog Horm Res 55: 15-35, 2000.

7. Gerber HP, Vu TH, Ryan AM, Kowalski J, Werb Z and Ferrara N: VEGF couples hypertrophic cartilage remodeling, ossification and angiogenesis during endochondral bone formation. Nat Med 5: 623-628, 1999.

8. Hedman M, Hartikainen J, Syvanne M, Stjernvall J, Hedman A, Kivela A, Vanninen E, Mussalo H, Kauppila E, Simula S, et al: Safety and feasibility of catheter-based local intracoronary vascular endothelial growth factor gene transfer in the prevention of postangioplasty and in-stent restenosis and in the treatment of chronic myocardial ischemia: phase II results of the Kuopio Angiogenesis Trial (KAT). Circulation 107: 2677-2683, 2003.

9. Bruder SP and Fox BS: Tissue engineering of bone. Cell based strategies. Clin Orthop Relat Res (Suppl): S68-S83, 1999.

10. Song L, Webb NE, Song Y and Tuan RS: Identification and functional analysis of candidate genes regulating mesenchymal stem cell self-renewal and multipotency. Stem Cells 24: 1707-1718, 2006.

11. Gronthos S, Akintoye SO, Wang CY and Shi S: Bone marrow stromal stem cells for tissue engineering. Periodontol 41: 188-195, 2006.

12. Turgeman G, Pittman DD, Muller R, Kurkalli BG, Zhou S, Pelled G, Peyser A, Zilberman Y, Moutsatsos IK and Gazit D: Engineered human mesenchymal stem cells: a novel platform for skeletal cell mediated gene therapy. J Gene Med 3: 240-251, 2001.

13. Spector JA, Mehrara BJ, Greenwald JA, Saadeh PB, Steinbrech DS, Bouletreau PJ, Smith LP and Longaker MT: Osteoblast expression of vascular endothelial growth factor is modulated by the extracellular microenvironment. Am J Physiol Cell Physiol 280: C72-C80, 2001.

14. Henrich D, Hahn P, Wahl M, Wilhelm K, Dernbach E, Dimmeler S and Marzi I: Serum derived from multiple trauma patients promotes the differentiation of endothelial progenitor cells in vitro: possible role of transforming growth factor-betal and vascular endothelial growth factor165. Shock 21: 13-16, 2004.

15. Nussenbaum B, Teknos TN and Chepeha DB: Tissue engineering: the current status of this futuristic modality in head neck reconstruction. Curr Opin Otolaryngol Head Neck Surg 12: 311-315, 2004.

16. Morizono K, de Ugarte DA, Zhu M, Zuk P, Elbarbary A, Ashjian P, Benhaim P, Chen IS and Hedrick MH: Multilineage cells from adipose tissue as gene delivery vehicles. Hum Gene Ther 14: 59-66, 2003.

17. Niyibizi C, Baltzer A, Lattermann C, Oyama M, Whalen JD, Robbins PD and Evans $\mathrm{CH}$ : Potential role for gene therapy in the enhancement of fracture healing. Clin Orthop Relat Res S148-S153, 1998.

18. Midy V and Plouet J: Vasculotropin/vascular endothelial growth factor induces differentiation in cultured osteoblasts. Biochem Biophys Res Commun 199: 380-386, 1994.

19. Mayer H, Bertram H, Lindenmaier W, Korff T, Weber H and Weich H: Vascular endothelial growth factor (VEGF-A) expression in human mesenchymal stem cells: autocrine and paracrine role on osteoblastic and endothelial differentiation. J Cell Biochem 95: 827-839, 2005.

20. Geiger F, Bertram H, Berger I, Lorenz H, Wall O, Eckhardt C, Simank HG and Richter W: Vascular endothelial growth factor gene-activated matrix (VEGF165-GAM) enhances osteogenesis and angiogenesis in large segmental bone defects. J Bone Miner Res 20: 2028-2035, 2005.

21. Niida S, Kondo T, Hiratsuka S, Hayashi S, Amizuka N, Noda T, Ikeda $\mathrm{K}$ and Shibuya M: VEGF receptor 1 signaling is essential for osteoclast development and bone marrow formation in colony-stimulating factor 1-deficient mice. Proc Natl Acad Sci USA 102: 14016-14021, 2005.

22. Kxuyt MC, de Bruijn JD, Yuan H, van Blitterswijk CA, Verbout AJ, Oner FC and Dhert WJ: Optimization of bone tissue engineering in goats: a peroperative seeding method using cryopreserved cells and localized bone formation in calcium phosphate scaffolds. Transplantation 77: 359-365, 2004. 